\title{
Multiline treatment combining apatinib with toptecan for platinum-resistant recurrent ovarian cancer patients: a report of three cases
}

This article was published in the following Dove Press journal:

OncoTargets and Therapy

\section{Yuan Cheng \\ Juan Zhang \\ Haiyun Geng \\ Shukui Qin \\ Haiqing Hua}

Department of Medical Oncology, People's Liberation Army Cancer Center, Bayi Hospital Affiliated

Nanjing University of Chinese

Medicine, Nanjing, China
Correspondence: Haiqing Hua Department of Medical Oncology, People's Liberation Army Cancer Center, Bayi Hospital Affiliated Nanjing University of Chinese Medicine, No. 34 Yanggongjing Road, Qinhuai District, Nanjing, Jiangsu 21002, China Email 1214856875@qq.com

\begin{abstract}
The aim of this study was to observe the efficacy and safety of apatinib combined with toptecan therapy in the multiline treatment of platinum-resistant recurrent ovarian cancer patients. The clinical records of three patients with platinum-resistant recurrent ovarian cancer treated with apatinib combined with toptecan therapy were analyzed and followed up for 3 months, and the related literatures were reviewed. The three patients achieved partial response and the tumor marker CA125 levels decreased significantly as an outcome of the treatment. Major adverse reactions were hypertension, hand-foot skin reaction, and anemia, which were manageable with medication. Apatinib combined with toptecan multiline therapy in the treatment of platinum-resistant recurrent ovarian cancer patients is effective, and the adverse effects are tolerated. Large-scale studies should be conducted to further determine the efficacy and safety of this treatment protocol.
\end{abstract}

Keywords: ovarian cancer, apatinib, topotecan, gynecological oncology

\section{Introduction}

Ovarian cancer is one of the most common gynecologic malignant tumors. According to the latest report of Cancer Incidence and Mortality in China in 2013, National Central Cancer Registry released the number of newly added ovarian cancers in China as about 50 million in 2013, which is the tenth incidence in gynecologic malignant tumors. ${ }^{1}$ The World Health Organization indicates that more than 150,000 people in the world died of ovarian cancer in $2014 .^{2}$ Although the clinical efficacy of ovarian cancer treatment continues to improve, the recurrence rate of patients receiving standard treatment is $\sim 70 \%-80 \%$, and the 5-year survival rate of patients with advanced ovarian cancer is only 25\%-30\%. ${ }^{3}$ Topotecan (Xinze ${ }^{\circledR}$, RuiNian Best Company, Nanjing, China) represents one of the best studied therapeutics in platinum-resistant ovarian cancer. ${ }^{4}$ The growth of tumors is dependent on oxygen and nutrients provided by new blood vessels. Apatinib (AiTan ${ }^{\circledR}$, JiangSu HengRui Medical Co. Ltd., Jiangsu, China) can effectively inhibit the formation of tumor vessels. So we tried to combine the two drugs in the treatment of advanced ovarian cancer. Here, we report three encouraging cases using apatinib combined with topotecan to treat platinum-resistant recurrent ovarian cancer in our hospital.

\section{Ethics approval}

Written informed consent has been provided by the patients to have the case details and any accompanying images published. 


\section{Case series}

\section{Case I}

Patient I, female, 62 years old, presented with a tumor. On November 20, 2012, abdominal computed tomography (CT) showed bilateral ovarian masses and elevated CA125 level, one of the tumor markers of ovarian cancer, of $1,578 \mathrm{U} / \mathrm{mL}$. She underwent total abdominal hysterectomy, bilateral adnexectomy, partial omentectomy, and resection of pelvic tumor nodules on January 25, 2013. The postoperative pathological analysis indicated that it was a case of grade I-II bilateral ovarian serous papillary cystadenocarcinoma, which originated from a $10 \times 10 \times 5.5 \mathrm{~cm}^{3}$ left ovarian tumor and a $5.5 \times 5 \times 2 \mathrm{~cm}^{3}$ right ovarian tumor (T2bN0M0). No residual lesions were detected in imaging. The patient was administered six cycles of paclitaxel/carboplatin (TC) regimen for adjuvant chemotherapy from December 2012 to July 2013. Her CA125 level returned to normal level in March 2013. In April 2015, positron emission tomography computed tomography (PET-CT) showed a pelvic mass, anterior mediastinal, parahepatic, retroperitoneal, and suprapancreatic lymph nodes, intrahepatic and parahepatic nodules and masses, right pleural thickening with multiple nodules, which increased fludeoxyglucose (FDG) metabolism. According to the imageological examination, the tumor appeared to have metastasized. Simultaneously, CA125 level reached $220 \mathrm{U} / \mathrm{mL}$. The patient was administered six cycles of paclitaxel/cisplatin (TP) chemotherapy regimen with bevacizumab from April 2015 to September 2015. The best therapeutic outcome achieved during the treatment was stable disease (SD). CA125 level returned to normal after the end of treatment. In March 2016, CT showed multiple nodular lesions in the right lung and right pleura, which were deemed metastatic. Simultaneously, CA125 level rose to $385 \mathrm{U} / \mathrm{mL}$. The patient was administered nine cycles of chemotherapy with etoposide. The best therapeutic outcome achieved during treatment was SD and CA125 level decreased to $51 \mathrm{U} / \mathrm{mL}$. In December 2016, CT showed soft tissue tumors enlarged in the pelvis and CA125 level was elevated to 138.7 U/mL, together indicating that the disease progressed. From December 2016 to January 2017, three cycles of GX chemotherapy were given. The best therapeutic outcome achieved was SD during the treatment. In February 2017, CT showed enlarged pelvic lesions, stable liver lesions, and enlarged retroperitoneal lymph nodes. The therapeutic evaluation indicated progressive disease (PD). CA125 level was 70.9 U/mL. From February 18, 2017, the patient received topotecan $\left(1.2 \mathrm{mg} / \mathrm{m}^{2}\right.$, oral [po], on days 1,8 , and 15 , every 4 weeks per cycle) combined with apatinib $(250 \mathrm{mg}$, po, once a day [qd]) for treatment. We attempted to increase the dose of apatinib (to $500 \mathrm{mg}$, qd) for 5 days starting from the second week. Following the increased doses, the patient developed grade 2 abdominal pain and we had to interrupt apatinib. Five days later, the pain subsided. We returned to the original dose (250 $\mathrm{mg} \mathrm{qd})$ and the patient did not report abdominal pain. Other adverse reactions included grade 1 proteinuria and grade 3 hypertension. These symptoms improved after treatment. In April 2017, CT showed that pelvic lesions, liver lesions, and retroperitoneal metastatic lymph nodes had all downsized compared with those prior to treatment (Figures 1 and 2). Also, CA125 returned to normal level. The therapeutic evaluation indicated partial response (PR). In January 2018, CT showed the progress of liver lesions
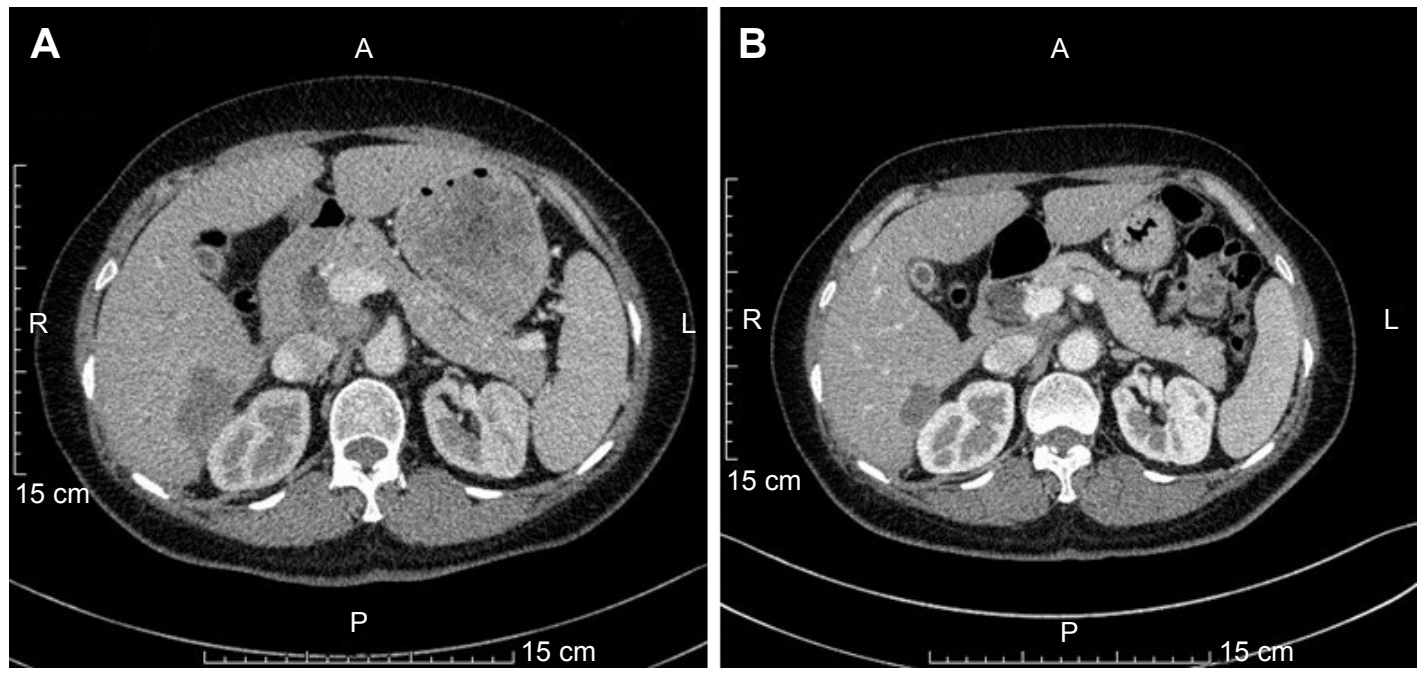

Figure I The tumor in the right posterior lobe of the liver is smaller than before. (A) Pretreatment. (B) Posttreatment. Abbreviations: $A$, anterior; $L$, left; $P$, posterior; $R$, right. 

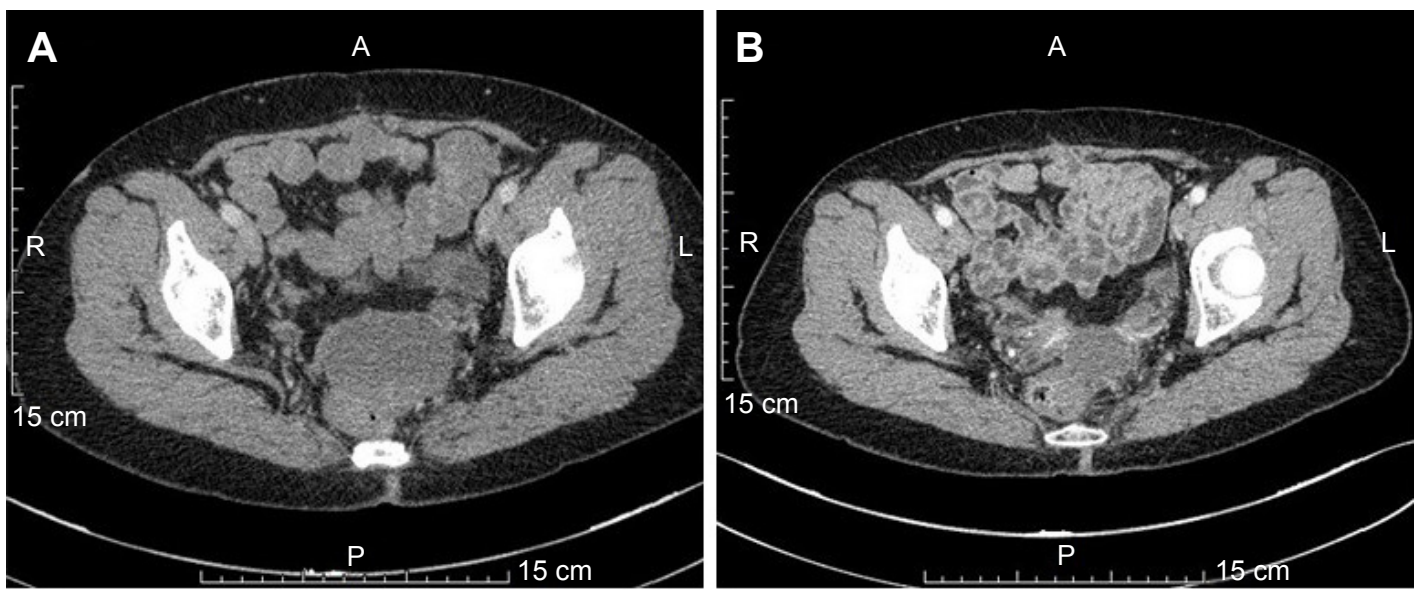

Figure 2 The tumors in the pelvic are smaller after treatment than before treatment. (A) Pretreatment. (B) Posttreatment. Abbreviations: $A$, anterior; $L$, left; $P$, posterior; $R$, right.

and new enlarged lymph nodes in the right supraclavicular fossa, together indicating that the disease progressed. The patient was administered 10 cycles of chemotherapy with topotecan, and time to progress (TTP) was 11 months. The patient is still alive.

\section{Case 2}

Patient II, female, 48 years old, presented with a tumor. On May 29, 2014, abdominal CT showed multiple spaceoccupying lesions in the pelvis, with the largest measuring $7.7 \times 7.4 \times 6.9 \mathrm{~cm}^{3}$ and elevated CA125 level of $1,191 \mathrm{U} / \mathrm{mL}$. On June 3, 2014, the patient underwent cytoreductive surgery (total abdominal hysterectomy + bilateral adnexectomy + omentectomy). No residual lesions were detected in postoperative imaging. The patient was administered six cycles of chemotherapy with docetaxel combined with oxaliplatin from July 2014 to October 2014 and her CA125 level returned to normal in October 2014. In June 2015, PET-CT showed that the FDG uptake of the nodules by the right iliac vascular region in the pelvis increased and was deemed metastatic; the FDG uptake of the lymph nodes by the abdominal aorta also increased and was deemed metastatic. CA125 level increased to $91.1 \mathrm{U} / \mathrm{mL}$. The patient was administered six cycles of TP chemotherapy regimen from June 2015 to October 2015. The best therapeutic evaluation was PR during the treatment. Also, CA125 level returned to normal during the treatment. In February 2016, CT showed that the lesions near right iliac vessels had enlarged, and CA125 level rose to $172 \mathrm{U} / \mathrm{mL}$. The patient underwent gamma knife radiotherapy for right iliac vascular lesions. After the treatment, the lesion regressed while CA125 level returned to normal. In October 2016, CT showed that the lesions in the right iliac vessels had enlarged while her CA125 level increased to $130.2 \mathrm{U} / \mathrm{mL}$. It was deemed PD. The patient was administered two cycles of GX chemotherapy from October 2016 to November 2016. After that, CT showed that the lesions of the right iliac vessels and the anterior lymph nodes of the abdominal aorta had enlarged; therefore, the tumor was deemed to have metastasized. The therapeutic evaluation was PD. The patient received gamma knife radiotherapy for abdominal aortic lymph node in December 2016. In March 2017, CT showed a left adnexal nodule and that multiple retroperitoneal and bilateral iliac vascular and left supraclavicular lymph nodes had enlarged and increased in number. CA125 level reached $315.5 \mathrm{U} / \mathrm{mL}$. Because of disease progression, from March 15, 2017, the patient was administered topotecan $\left(1.2 \mathrm{mg} / \mathrm{m}^{2}\right.$, po, days 1,8 , and 15 , every 4 weeks a cycle) combined with apatinib $(250 \mathrm{mg}$, po, qd) for treatment. We tried to increase the dose of apatinib (to $500 \mathrm{mg}$, qd) for 7 days starting from the second week. As a result of the increased dose, the patient developed grade 3 hand-foot skin reaction, with main symptom of multiple blisters and ulceration on bilateral palms and soles. As her pain significantly reduced her ability to walk, we decided to reduce the dosage of apatinib to $250 \mathrm{mg}$ /day to continue treatment and also gave her some urea cream to treat the skin reaction. The hand-foot skin reaction subsided after 1 week. In April 2017, CT showed that the lesions of bilateral adnexal nodules had downsized, and the systemic lymph nodes were slightly smaller than before (Figure 3), while CA125 level decreased to $75.9 \mathrm{U} / \mathrm{mL}$. The therapeutic evaluation was PR. In November 2017, CT showed liver lesion, adnexal nodular lesion, and multiple lymph node had enlarged, together indicating that the disease progressed. The patient was administered seven cycles of chemotherapy with topotecan, and TTP was 8 months. The patient is still alive. 

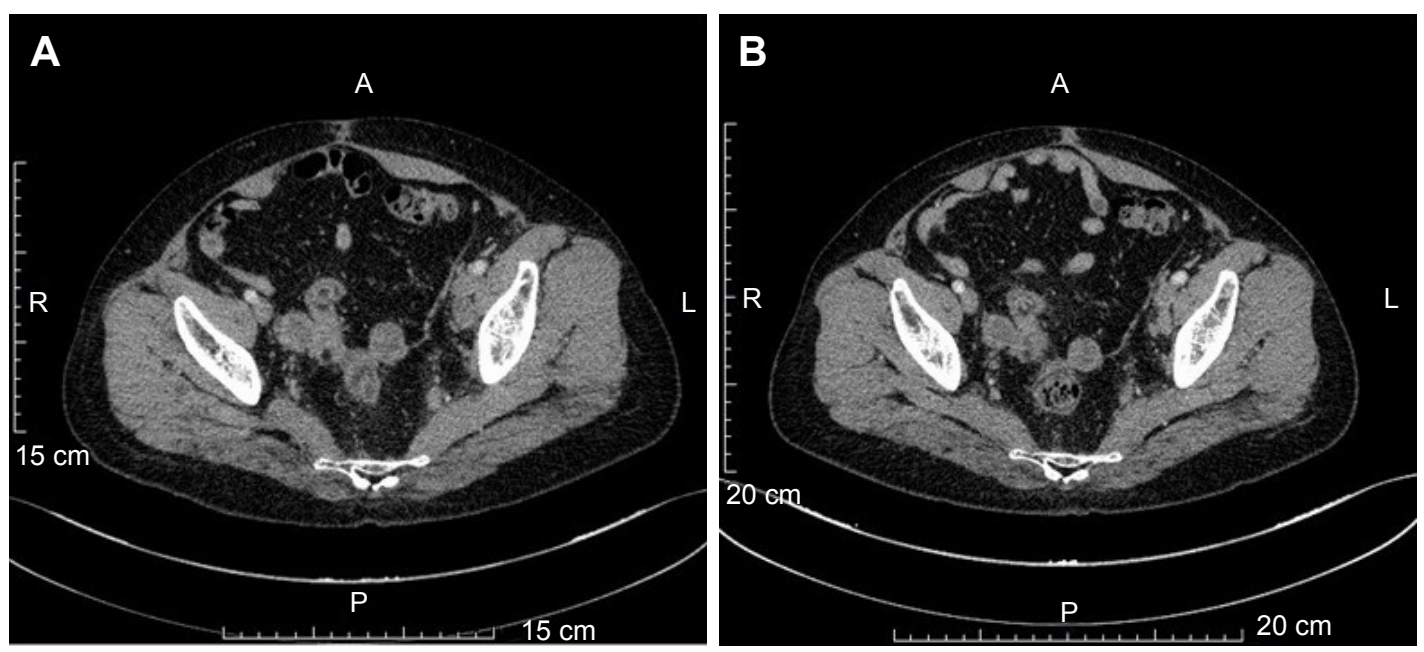

Figure 3 The tumors in the pelvic are smaller after treatment than before treatment. (A) Pretreatment. (B) Posttreatment. Abbreviations: $A$, anterior; $L$, left; $P$, posterior; $R$, right.

\section{Case 3}

Patient III, female, 35 years old, presented with a tumor. A pelvic mass was discovered as a result of a physical examination in December 2013; the diameter was about $15 \mathrm{~cm}$ and her CA125 level reached $365.7 \mathrm{U} / \mathrm{mL}$. She underwent total abdominal hysterectomy, bilateral adnexectomy, partial omentectomy, and pelvic lymph node dissection, abdominal aortic lymph node dissection on December 26, 2014. Intraoperatively, we noticed medium pale yellow ascites in abdominal pelvic cavity, left ovarian cystic tumors (about $14 \times 10 \times 8.0 \mathrm{~cm}^{3}$ ), and right tubal cystic tumor (about $7 \times 6 \times 5.0 \mathrm{~cm}^{3}$ ). Also, the surfaces of these tumors were all intact. No lesions can be found in pelvic, and none was detected on the surface of the intestine, omentum, abdominal wall, stomach, liver, spleen, and other organs. No malignant tumor cells were found in ascites. Postoperative pathology confirmed the diagnosis of left ovary clear-cell carcinoma. There was no tumor infiltration on the left fallopian tube, right ovary, bilateral fallopian tubes, omentum, and no metastatic lymph nodes were seen. The postoperative staging was IA. The patient was administered six cycles of adjuvant chemotherapy with TC (paclitaxel + carboplatin) regimen from January 24, 2015, to June 12, 2015. CA125 level returned to normal in April 2015. On December 15, 2015, abdominal CT showed cystic solid masses in pelvis, which were deemed metastatic. On December 25, 2015, pelvic tumor resection was performed, and postoperative pathology showed poorly differentiated carcinoma, which was metastasis originating from ovarian clear-cell carcinoma. On February 2, 2016, CT scan showed right pelvic nodules, multiple circular low-density tumors of both the left outer lobe and the right lobe of liver, which were deemed metastatic. At that time, CA125 level decreased to $38 \mathrm{U} / \mathrm{mL}$. The patient was administered nine cycles of chemotherapy with FOLFIRI regimen from February 3, 2016, to June 10, 2016. The best outcome achieved during the treatment was SD. Also, on April 1, 2017, CA125 level returned to normal during the treatment period. On June 17, 2016, CT showed more advanced liver lesions. The patient was administered two cycles of chemotherapy with GEMOX regimen from July 2016 to August 2016. Also, after that, no antitumor therapy was administered. On March 9, 2017, CT showed multiple metastases in the liver, spleen, abdomen, and pelvis. Abdominal mass resection was performed on April 1, 2017. Postoperative pathology confirmed the diagnosis of ovary clear-cell carcinoma metastasis. On April 26, 2017, CT showed multiple metastasis of the liver, spleen, abdominal, and pelvic, indicating tumor progress (compared with March 9, 2017), while CA125 level rose to $299.5 \mathrm{U} / \mathrm{mL}$. From May 10, 2017, the patient received topotecan $\left(1.2 \mathrm{mg} / \mathrm{m}^{2}\right.$, po, days 1,8 , and 15 , every 4 weeks a cycle) combined with apatinib (250 mg, po, qd) for treatment. In June 2017, CT reexamination demonstrated that liver, spleen, abdominal, and pelvic peritoneum multiple metastases had regressed noticeably (Figure 4), while CA125 level decreased to $91.5 \mathrm{U} / \mathrm{mL}$. The therapeutic evaluation was PR. The adverse event (AE) was mainly grade 3 anemia, which recovered well after blood transfusion. No other side effects were found. In October 2017, CT showed that the multiple metastasis lesions of the liver, spleen, abdominal, and pelvic had enlarged, together indicating that the disease progressed. The patient was administered five cycles of chemotherapy with topotecan, and TTP was 5 months. The patient is still alive.

\section{Discussion}

Ovarian cancer has some of the highest incidences and mortality rates among gynecological cancers. Most patients are 

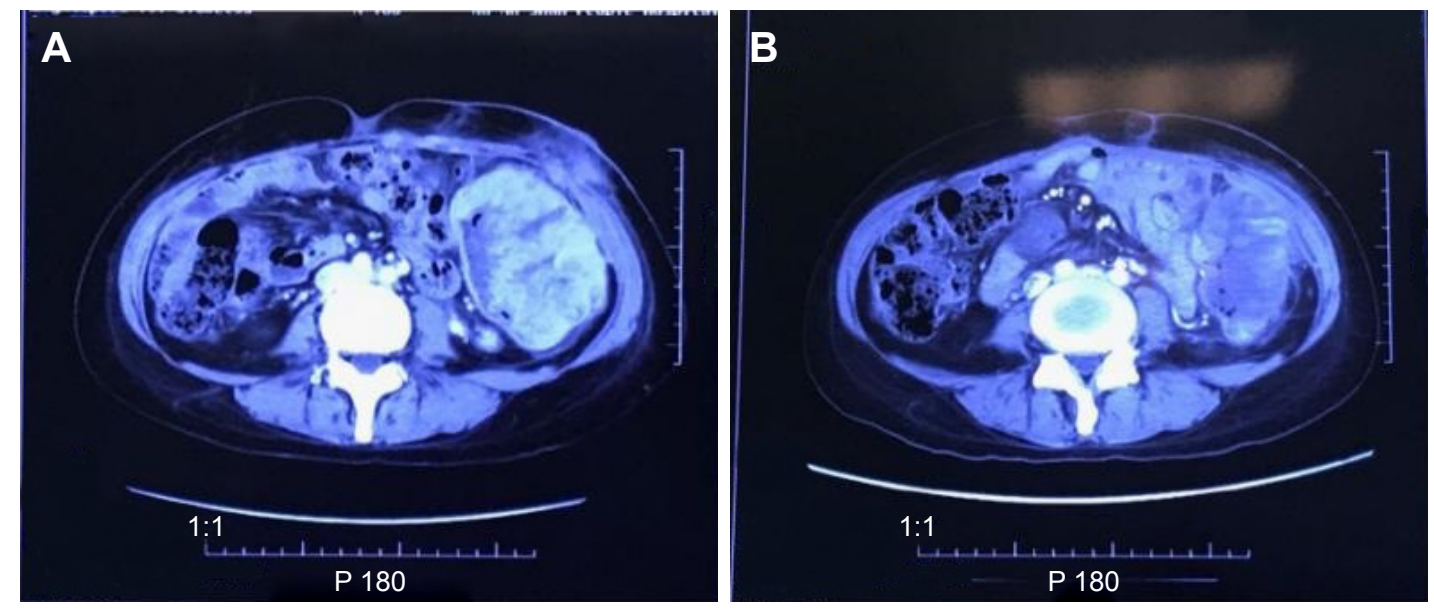

Figure 4 The tumors in the abdominal cavity are smaller after treatment than before treatment. (A) Pretreatment. (B) Posttreatment.

diagnosed at advanced stages. After cytoreductive surgery, systemic treatment is particularly important.

National Comprehensive Cancer Network guideline for ovarian cancer (2017, Version 1.0) recommends carboplatin plus paclitaxel as the standard chemotherapy regimen for initial treatment. Recurrent ovarian cancer is further divided into two types: platinum resistance and platinum sensitive ${ }^{5-8}$ Platinum-based chemotherapy is the first choice for platinum-sensitive ovarian cancer patients. Non-platinum monochemotherapy is the first choice for platinum-resistant recurrent ovarian cancer patients. The guideline recommends the following cytotoxic agents: docetaxel (response rate of $22 \%$ ), gemcitabine (response rate of $19 \%$ ), topotecan (response rate of 20\%), liposomal doxorubicin (response rate of $26 \%$ ), oral etoposide (response rate of $27 \%$ ), and paclitaxel (response rate of $21 \%)^{9}$

Targeted drugs have also demonstrated greater efficacy in the treatment of ovarian cancer. Bevacizumab was the first targeted drug indicated for platinum-resistant recurrent ovarian cancer approved by the US Food and Drug Administration. Two Phase III clinical studies (GOG0218 and ICON7) using bevacizumab plus carboplatin/paclitaxel to treat advanced ovarian cancer patients as first-line therapy and mono-bevacizumab as post-chemotherapy maintenance therapy showed that the disease progression-free survival (PFS) was significantly prolonged. ${ }^{10-12}$ In addition, two poly (ADP-ribose) polymerase inhibitors (olaparib and rucaparib) have also been approved for advanced ovarian cancer. ${ }^{13,14}$ Pazopanib is an oral multikinase inhibitor of c-KIT, fibroblast growth factor receptor (FGFR), plateletderived growth factor receptor (PDGFR), and vascular endothelial growth factor receptor (VEGFR), which is recommended as the maintenance therapy for patients with stages II-IV epithelial ovarian cancer. ${ }^{15}$ Bevacizumab is the only targeted therapy for ovarian cancer approved in China.

Apatinib is a novel highly selective small-molecule oral inhibitor of VEGFR-2 developed in China. As an antiangiogenesis agent, it effectively inhibits the proliferation, migration, and angiogenesis of vascular endothelial cells. Apatinib is currently approved by the Chinese State Food and Drug Administration for the treatment of advanced gastric adenocarcinoma or gastroesophageal junction adenocarcinoma patients in the third-line or latter settings. The drug has notable antitumor effects in various tumor models, such as colon, gastric, lung, and liver cancers. ${ }^{16}$ Currently, a number of Phase II/III trials are ongoing to assess its efficacy in gastric, lung, esophageal, liver, thyroid, osteosarcoma cancers, and other tumors. The designs include mono or combination therapies. ${ }^{17}$ Clinical efficacy of apatinib in various malignant tumors in second-line or latter settings has also been reported. There are also some case reports of apatinib being used to treat gynecological cancers. ${ }^{18-20}$

In this report, patient I progressed three times. She received four chemotherapy regimens over a total of 24 treatment cycles during which she also received bevacizumab as a combination therapy for 6 months. Patient II has progressed four times. She received three chemotherapy regimens over a total of 14 cycles of chemotherapy and two gamma knife radiotherapy to address her intraperitoneal nodules. Patient III progressed four times. She received two chemotherapy regimens over a total of 11 cycles of chemotherapy and two pelvic tumor resections. When these three patients progressed again, there was no standard treatment. It was inappropriate to treat them with strong cytotoxic drugs considering their fitness levels and bone marrow functions. Available clinical literatures suggest few chemotherapy drugs for multiline latestage therapy with satisfactory results. The three patients had 
not received chemotherapy with singla topotecan before, so we decided to choose the one. It is reported that women with relapsed ovarian cancer may benefit from similar effectiveness but significantly lower hematologic toxicity if topotecan is administered in a weekly schedule. ${ }^{21,22}$ So we chose the weekly schedule.

The Phase II randomized multicenter clinical trial (MITO-11), which used weekly paclitaxel with or without pazopanib (a multikinase inhibitor of c-KIT, FGFR, PDGFR, and VEGFR) to treat platinum-resistant or platinum-refractory advanced ovarian cancer as a second-line therapy, showed a PFS of 3.5 months in the weekly paclitaxel arm and 6.3 months in the pazopanib arm and a hazard ratio of 0.42 (95\% CI, 0.25-0.69), $p=0.0002$. Median overall survival (OS) was 14.8 months in the weekly paclitaxel arm and 18.7 months in the pazopanib arm with a median follow-up period of 12.5 months. The conclusion is that paclitaxel combined with pazopanib significantly prolonged PFS and OS in the treatment of platinum-resistant or platinum-refractory advanced ovarian cancer patients. ${ }^{23}$ The mechanism of pazopanib is similar to apatinib, which has not been approved in China. There are also some positive reports about apatinib combined with chemotherapy in the treatment of advanced breast cancer patients in China. ${ }^{24}$

We chose apatinib combined with topotecan eventually. We chose topotecan capsules as a monotherapy administered weekly $\left(1.2 \mathrm{mg} / \mathrm{m}^{2}\right.$, oral, on days 1,8 , and 15 , every 4 weeks a cycle). We also administered low-dose apatinib $(250 \mathrm{mg}$, oral, qd) concurrently. After 8 weeks, the tumor sizes of all the three patients regressed, all indicating PR, and the tumor marker CA125 levels decreased significantly. These three cases show the potential of apatinib combined with chemotherapy in advanced ovarian carcinoma patients.

The common AEs of apatinib include hematological toxicity (leukopenia, neutropenia, thrombocytopenia, etc) and nonhematological toxicity (hypertension, proteinuria, hand-foot syndrome, fatigue, diarrhea, etc). Most AEs can be managed by dose reduction or interruption. The main AEs of the three patients were hand-foot syndrome, hypertension, anemia, loss of appetite, and fatigue, which were all relieved after appropriate treatment and dose reduction of apatinib.

Patient I had prior history of hypertension and had been taking oral valsartan and hydrochlorothiazide tablets for a long time, which retained her blood pressure in the normal range. Her blood pressure increased after taking the apatinib but was then controlled by nifedipine. Patient II developed grade 3 hand-foot skin reaction. The condition was relieved after dose reduction of the apatinib and localized treatment.
Patients III had prior history of grade 2 anemia and developed grade 3 anemia after taking apatinib. This later improved to grade 1 after blood transfusion. It is worth mentioning that patients I and II were stable after taking apatinib for the first week of treatment but developed AE after we increased the dose to $500 \mathrm{mg} /$ day starting from the second week. They developed grade 3 hypertension and grade 2 abdominal pain after increasing the dose, which proved too difficult for the patients to tolerate. We then reduced dosage or stopped apatinib, and eventually settled for a lower dosage of $250 \mathrm{mg} /$ day. The patients were then able to tolerate apatinib and continue treatment.

\section{Conclusion}

Apatinib combined with chemotherapy may potentially be effective in the treatment of advanced ovarian cancer patients in multiline therapies. However, large-scale clinical trials should be conducted to test this hypothesis in the future. We also need to assess the optimal drug combinations and dosage.

\section{Disclosure}

The authors report no conflicts of interest in this work.

\section{References}

1. Chen WQ, Zheng RS, Zhang SW, et al. Report of cancer incidence and mortality in China 2013. China Cancer. 2017;26(1):1-7.

2. Monk BJ, Poveda A, Vergote I, et al. Anti-angiopoietin therapy with trebananib for recurrent ovarian cancer (TRINOVA-1): a randomised, multicentre, double-blind, placebo-controlled phase 3 trial. Lancet Oncol. 2014;15(8):799-808.

3. Liu D, Ha C, Zhang X, Zhang Z, Liu P. Molecular implication of ADAM-15 and 17 in intrauterine adhesions. Eur J Obst Gynecol Reprod Biol. 2013;170(1):264-269.

4. Gordon AN, Tonda M, Sun S, Rackoff W; Doxil Study 30-49 Investigators. Long-term survival advantage for women treated with pegylated liposomal doxorubicin compared with topotecan in a phase 3 randomized study of recurrent and refractory epithelial ovarian cancer. Gynecol Oncol. 2004;95(1):1-8.

5. Iyengar TD, Herzog TJ. Management of symptomatic ascites in recurrent ovarian cancer patients using an intra-abdominal semipermanent catheter. Am J Hosp Palliat Care. 2002;19(1):35-38.

6. Brooks RA, Herzog TJ. Long-term semi-permanent catheter use for the palliation of malignant ascites. Gynecol Oncol. 2006;101(2):360-362.

7. Fung-Kee-Fung M, Oliver T, Elit L, Oza A, Hirte HW, Bryson P. Optimal chemotherapy treatment for women with recurrent ovarian cancer. Curr Oncol. 2007;14(5):195-208.

8. Parmar MK, Ledermann JA, Colombo N, et al; ICON and AGO Collaborators. Paclitaxel plus platinum-based chemotherapy versus conventional platinum-based chemotherapy in women with relapsed ovarian cancer: the ICON4/AGO-OVAR-2.2 trial. Lancet. 2003; 361(9375):2099-2106.

9. NCCN Clinical Practice Guideline in Oncology (NCCN Guidelines ${ }^{\circledR}$ ). Ovarian Cancer Including Fallopian Tube Cancer and Primary Peritoneal Cancer Version 4[EB/OL] [2017-04-12]. Available from: http://www. nccn.org/professionals/physician_gls/__guidelines.asp\#varian. Accessed November 7, 2017. 
10. Burger RA, Brady MF, Rhee J, et al. Independent radiologic review of the Gynecologic Oncology Group Study 0218, a phase III trial of bevacizumab in the primary treatment of advanced epithelial ovarian primary peritoneal, or fallopian tube cancer. Gynecol Oncol. 2013;131(1):21-26.

11. Burger RA, Brady MF, Bookman MA, et al; Gynecologic Oncology Group. Incorporation of bevacizumab in the primary treatment of ovarian cancer. N Engl J Med. 2011;365(26):2473-2483.

12. Perren TJ, Swart AM, Pfisterer J, et al. A phase 3 trial of bevacizumab in ovarian cancer. N Engl J Med. 2011;365:2484-2496.

13. Kaufman B, Shapira-Frommer R, Schmutzler RK, et al. Olaparib monotherapy in patients with advanced cancer and a germline BRCA1/2 mutation. J Clin Oncol. 2015;33(3):244-250.

14. Swisher EM, Lin KK, Oza AM, et al. Rucaparib in relapsed, platinumsensitive high-grade ovarian carcinoma (ARIEL2 Part 1): an international, multicentre, open-label, phase 2 trial. Lancet Oncol. 2017;18(1): 75-87.

15. du Bois A, Floquet A, Kim JW, et al. Incorporation of pazopanib in maintenance therapy of ovarian cancer. J Clin Oncol. 2014;32(30): 3374-3382.

16. Li X, Zhang C, Tan H, et al. The clinical effective observation of apatinib mesylate tablets in the treatment of advanced non-small cell lung cancer. Chin J Biochem Med. 2016;2:91-93.

17. Tu Yan, Peng Feng. Progress in clinical research of apatinib treatment of malignant tumor. Chin J Oncol. 2016;43(12):545-548.
18. Song J, Chen Y, Xu C, et al. The effect of apatinib treatment of 53 cases of advanced primary liver cancer. J Clin Pathol. 2017;37(3):557-563.

19. Li L, Zhang F, Nan F, et al. Apatinib treatment for refractory malignant tumor clinical observation of 43 cases. Chin J Pract Diagn Ther. 2017; 7: 594-596.

20. Peng Yu, Li Haitao, Lin Daren, et al. The clinical curative effect of apatinib treatment of three and more than three lines of advanced solid tumors. J Baotou Med Coll. 2017;33(3):8-16.

21. Sehouli J, Stengel D, Harter P, et al. Topotecan weekly versus conventional 5-day schedule in patients with platinum-resistant ovarian cancer: a randomized multicenter phase II trial of the North-Eastern German Society of Gynecological Oncology Ovarian Cancer Study Group. J Clin Oncol. 2011;29(2):242-248.

22. Herzog TJ, Sill MW, Walker JL, et al. A phase II study of two topotecan regimens evaluated in recurrent platinum-sensitive ovarian, fallopian tube or primary peritoneal cancer: a Gynecologic Oncology Group Study (GOG 146Q). Gynecol Oncol. 2011;120(3):454-458.

23. Pignata S, Lorusso D, Scambia G, et al; MITO 11 Investigators. Pazopanib plus weekly paclitaxel versus weekly paclitaxel alone for platinum-resistant or platinum-refractory advanced ovarian cancer (MITO 11): a randomised, open-label, phase 2 trial. Lancet Oncol. 2015; 16(5):561-568.

24. Zhang Y, Xu H, Zhang F. Apatinib combined treatment of relapsed breast cancer: 2 cases report and literature review. Cancer. 2017;5:518-523.
OncoTargets and Therapy

\section{Publish your work in this journal}

OncoTargets and Therapy is an international, peer-reviewed, open access journal focusing on the pathological basis of all cancers, potential targets for therapy and treatment protocols employed to improve the management of cancer patients. The journal also focuses on the impact of management programs and new therapeutic agents and protocols on

\section{Dovepress}

patient perspectives such as quality of life, adherence and satisfaction. The manuscript management system is completely online and includes a very quick and fair peer-review system, which is all easy to use. Visit http://www.dovepress.com/testimonials.php to read real quotes from published authors. 